\title{
Rapid and Green Fabrication of Carbon Dots for Cellular Imaging and Anti-Counterfeiting Applications
}

\author{
Chen Li, Xiaoyan Sun, Yuan Li, Hailu Liu, Bibo Long, Dong Xie, Junjia Chen,* and Ke Wang*
}

Cite This: ACS Omega 2021, 6, 3232-3237

Read Online

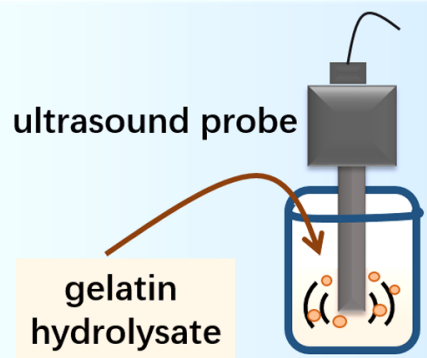

$30 \mathrm{~min}$

carbon dots

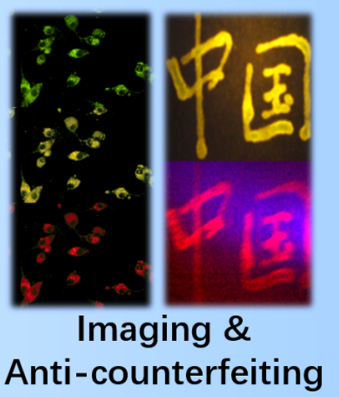

ABSTRACT: Carbon dots (CDs) with plenty of favorable properties have been extensively investigated in diverse areas including bioimaging, biomedicine, sensor, energy storage, anti-counterfeiting, photocatalysis, and optoelectronic devices. Herein, a simple, rapid, and green sonochemical-assisted method for fabricating nitrogen-doped CDs has been developed. In this approach, the nitrogen-doped CDs can be obtained through irradiation by intensive ultrasonic waves from ultrasonic probes in 30 min. The achieved CDs exhibit excellent water dispersibility, which can be ascribed to their high functionalization. Importantly, the CDs also demonstrate remarkable fluorescent properties, high photostability, and low cytotoxicity, which can be utilized for multicolor cellular imaging and anti-counterfeiting applications. As far as we know, the sonochemical-assisted method for rapidly synthesizing nitrogendoped CDs from gelatin has never been reported before. Significantly, the sonochemical-assisted approach to rapidly fabricate CDs is versatile for the facile construction of fluorescent CDs, and the obtained CDs can be potentially used in various areas including bioimaging and anti-counterfeiting.

\section{INTRODUCTION}

Carbon dots (CDs) have aroused keen interest with their plenty of favorable properties, such as tunable photoluminescence, splendid photostability, high biocompatibility, and electronic transport properties, which made CDs play significant roles in various fields including bioimaging, biomedicine, sensor, energy storage, anti-counterfeiting, photocatalysis, and optoelectronic devices. ${ }^{1-7}$ To date, various approaches have emerged to construct CDs, and these methods can be generally sorted as "top-down" and "bottomup" strategies according to their carbon sources. ${ }^{5-8}$ The topdown methods usually would smash bulk carbon sources into smaller units, which were limited by their expensive equipment and high consumption of energy. ${ }^{9}$ On the other hand, the bottom-up approaches always employed a more facile and simple manner, such as hydrothermal treatment, pyrolysis, and chemical oxidation. ${ }^{10-13}$ In recent years, many new techniques have constantly emerged to construct CDs, which revealed impressive superiorities in their high efficiency and low consumption. $^{14-19}$

Gelatin, a natural polymer, is the product of partial hydrolysis of collagen, which can be divided into photographic gelatin, edible gelatin, and industrial gelatin according to their properties and uses. In recent decades, due to its extensive sources, great biocompatibility, and outstanding biodegradability, gelatin demonstrates excellent performance in the fields of biomaterials, such as drug carriers, artificial skin, and scaffolds for tissue engineering. ${ }^{20-22}$ Importantly, gelatin can also be employed to fabricate CDs with all these superiorities. $\mathrm{Li}$ and co-workers reported their work on the preparation of luminescent CDs through a hydrothermal process from gelatin. ${ }^{23}$ Wang and co-workers synthesized CDs/gelatin composite by a microwave approach. ${ }^{24}$ Nevertheless, it is

Received: November 22, 2020

Accepted: December 29, 2020

Published: January 21, 2021

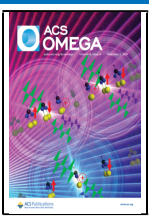


time-consuming to achieve mass production of CDs through hydrothermal treatment, and the CDs/gelatin composite obtained from the microwave method was not suitable for some applications in which only CDs were needed.

In this work, we described the rapid fabrication of nitrogendoped CDs from gelatin via a sonochemical-assisted green method. Compared with the ultrasound bath, the ultrasound probe demonstrated higher efficiency and effectiveness in the preparation of CDs. As revealed in Figure 1, with the same

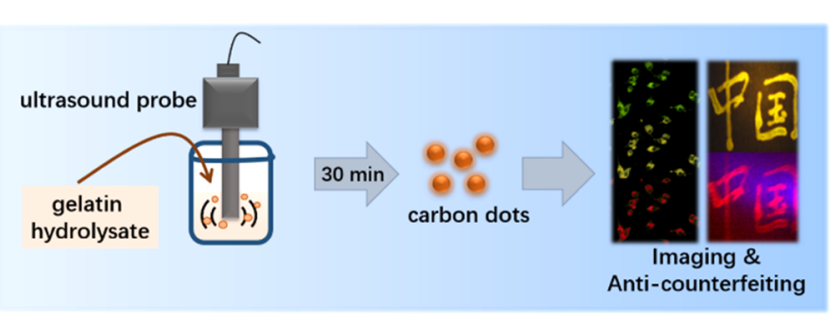

Figure 1. Schematic representation showing the rapid fabrication of $\mathrm{CDs}$ through a sonochemical-assisted approach with an ultrasound probe and their fluorescence imaging and anti-counterfeiting applications.

ultrasonic frequency and irradiation time, fluorescent CDs can be obtained via the ultrasonic probe strategy, while only some larger polymer nanoparticles were achieved in the ultrasonic bath method. The obtained CDs were prepared from gelatin through simple processing in water without adding any catalyst, acid, or alkali solvents. Moreover, the CDs exhibited excellent water dispersibility, splendid biocompatibility, and remarkable fluorescent properties, which were promising for bioimaging and anti-counterfeiting applications. ${ }^{25-27}$ As far as we know, the sonochemical-assisted method for rapidly synthesizing nitrogen-doped CDs from gelatin has never been reported before. Significantly, the sonochemical-assisted approach to rapidly fabricate CDs is versatile for the facile construction of fluorescent CDs from many analogous carbon sources.

\section{RESULTS AND DISCUSSION}

2.1. Rapid Fabrication of Nitrogen-Doped CDs from Gelatin. The CDs were constructed via a green sonochemical approach from gelatin, as illustrated in Figure 1. Gelatin was hydrolyzed by papain and then irradiated by intensive ultrasonic waves for $30 \mathrm{~min}$ using ultrasonic probes. After further routine centrifugation and lyophilization, the nitrogendoped CDs were achieved. The sonochemical method is rapid, green, and facile, with no harsh conditions or complicated manipulations. Importantly, the obtained nitrogen-doped CDs could demonstrate stable photoluminescence and low cytotoxicity. As a contrast, gelatin was also put in an ultrasonic bath without probes for irradiation at the same time. However, there were few CDs in the product but some larger polymer nanoparticles (PNs). The detailed characterizations would be discussed later.

In this method, it was the cavitation phenomena generated from the intensive ultrasonic waves that can be used to explain the formation of CDs. With the alternative high pressure and low pressure in the liquid medium, many microbubbles would rapidly form, grow, and collapse, which would lead to the formation of special hot spots with high temperatures of over $5000 \mathrm{~K}$, pressures of more than $1000 \mathrm{~atm}$, and heating and cooling rates greater than $10^{10} \mathrm{Ks}^{-1} \cdot{ }^{16}$ It is believed that the condensation and carbonization of gelatin happened during the above acoustic cavitation. ${ }^{5}$ Nevertheless, the clear mechanism of CDs formation has not been generally agreed till the present moment. However, it can be inferred from the experiments that, with the same frequency and irradiation time, the carbonization energy for treating gelatin in the ultrasonic bath was not enough to prepare CDs. Excitingly, in our strategy, the CDs could be rapidly prepared via the sonochemical-assisted green method.

2.2. Characterization of CDs. To assess the morphology of the achieved nitrogen-doped CDs, high-resolution transmission electron microscopy (HR-TEM) was employed. As revealed in Figure 2A, the CDs showed a spherical shape and
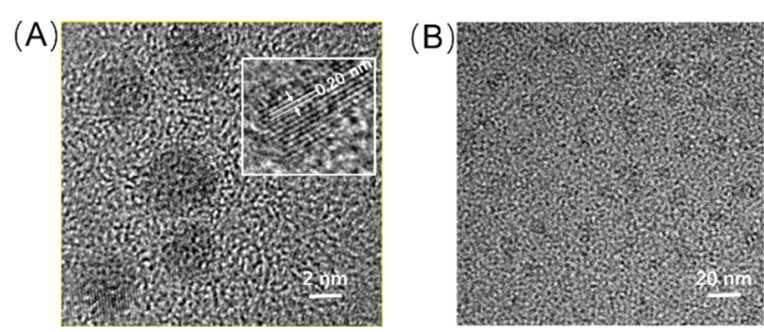

Figure 2. (A) HR-TEM image of obtained CDs through an ultrasonic probe approach and the inset showed their crystalline fringe with a regular lattice spacing of $0.18 \mathrm{~nm}$. (B) HR-TEM image of obtained PNs via the ultrasound bath method.

were with a mean diameter of $3.8 \mathrm{~nm}$. Clearly, the inset showed their crystalline fringe, and the regular lattice spacing is $0.18 \mathrm{~nm}$, which remains well with the (102) diffraction planes of $\mathrm{sp}^{2}$ graphitic carbon. ${ }^{13}$ These results can prove that the CDs had been successfully prepared via the probe-assisted sonochemical approach. The morphonology of PNs was also investigated, as shown in Figure 2B. Nevertheless, the PNs were larger than CDs with a mean diameter of $10.2 \mathrm{~nm}$, and no crystalline fringes can be detected in these nanoparticles with irregular shapes. It was reckoned that the condensation of gelation had also taken place during the sonochemical method without probes, but the next carbonization procedure was not finished yet.

The chemical compositions of the achieved CDs were measured by Fourier transform infrared spectra (FTIR) together with X-ray photoelectron spectra (XPS) analysis. As exhibited in Figure 3, the characteristic absorption peak at $3293 \mathrm{~cm}^{-1}$ is from $\mathrm{O}-\mathrm{H} / \mathrm{N}-\mathrm{H}, 1631 \mathrm{~cm}^{-1}$ is for the stretching vibration band of $\mathrm{C}=\mathrm{O}$, and 1080 and $874 \mathrm{~cm}^{-1}$ come from the stretching vibration band of $\mathrm{C}-\mathrm{O}$, which all indicate that the surface of $\mathrm{CDs}$ has been partially oxidized. $^{27,28}$ Furthermore, the obvious peak at $1540 \mathrm{~cm}^{-1}$

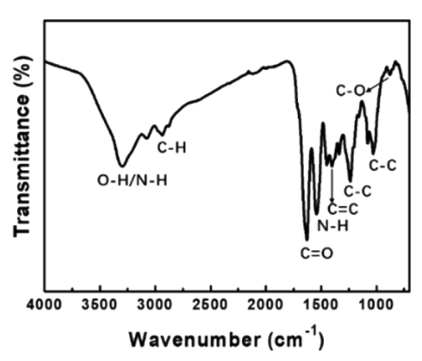

Figure 3. FTIR spectrum of obtained CDs. 
can be associated with $\mathrm{N}-\mathrm{H}$ bands of vibration and deformation, which manifested that there are some aminorelated groups on the CDs. Moreover, the absorption peaks at $1249 \mathrm{~cm}^{-1}$ can be ascribed to the symmetrical stretching vibration band of $\mathrm{C}-\mathrm{C}$, and the anti-symmetrical stretching vibration band of $\mathrm{C}-\mathrm{C}$ is seated at $1029 \mathrm{~cm}^{-1} .^{29,30}$ Additionally, the peaks at 2940 and $1405 \mathrm{~cm}^{-1}$ are assigned to the stretching vibrations $\mathrm{C}-\mathrm{H}$ and $\mathrm{C}=\mathrm{C}$, which further suggests the production of alkyl and aryl groups on their surface. ${ }^{31}$ Figure 4A shows the XPS data of CDs. The CDs are primarily

\section{(A)}
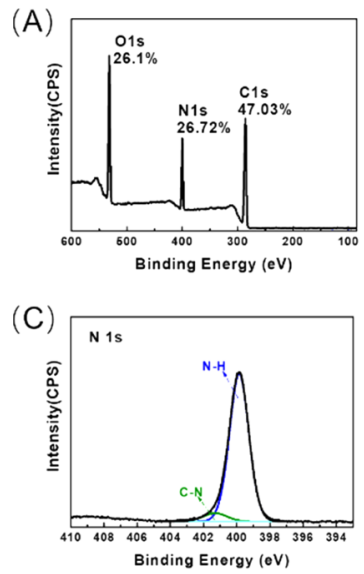

(B)

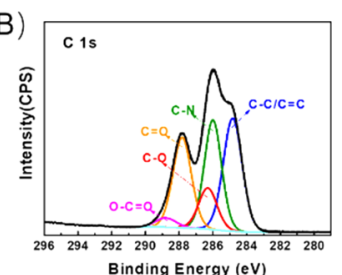

(D)

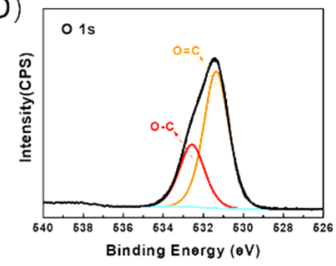

Figure 4. (A) XPS survey spectrum of CDs with detailed percent of different elements and high-resolution XPS scanning and their fitting curves of (B) C 1s, (C) N 1s, and (D) O 1s.

composed of carbon (47.03\%), oxygen (26.10\%), nitrogen $(26.72 \%)$, and sulfur $(0.16 \%)$. As revealed in Figure 4B, the high resolution of the $\mathrm{C} 1 \mathrm{~s}$ spectrum displays the peaks at $284.8,286.0,286.3,287.8$, and $288.8 \mathrm{eV}$, which indicates that there are $\mathrm{C}-\mathrm{C} / \mathrm{C}=\mathrm{C}, \mathrm{C}-\mathrm{N}, \mathrm{C}-\mathrm{O}, \mathrm{C}=\mathrm{O}$, and $\mathrm{O}-\mathrm{C}=\mathrm{O}$ groups functionalized on the surface of CDs. ${ }^{32}$ The functional groups endow the CDs with great solubility and stability in an aqueous system, which could also be further modified for diverse applications in different areas. Figure 4C and Figure 4D show the high resolution of $\mathrm{N} 1 \mathrm{~s}$ and $\mathrm{O}$ 1s spectra, respectively, which also prove the presence of amino-related functional groups and are consistent with the above FTIR results.

The detailed optical qualities of CDs were checked by investigating their UV-vis spectrum and fluorescence (FL) spectra. From Figure 4A, it can be observed that the CDs reveal a broad absorption band in the range of $300-400 \mathrm{~nm}$, which is attributed to the presence of CDs. The FL spectra in Figure $5 \mathrm{~A}$ reveal that the $\mathrm{CDs}$ could emit a fluorescence around $450 \mathrm{~nm}$ with an excitation wavelength of $365 \mathrm{~nm}$. Clearly, the inset photographs in Figure 5A show that the aqueous dispersion of CDs is transparent to daylight, whereas it demonstrates intense blue luminescence under UV light $\left(\lambda_{\max }=365 \mathrm{~nm}\right)$. As depicted in Figure 5B and Figure 5C, the $\mathrm{CDs}$ featured a typical photoluminescence property that the emission peaks would change with the variation of excitation wavelengths. As the excitation wavelengths changed from 300 to $500 \mathrm{~nm}$, their fluorescence band maximum would shift from 400 to $550 \mathrm{~nm}$. Also, the quantum yield of CDs was measured, and the detailed process can be seen in our earlier research. ${ }^{33}$ The result proves that the fluorescent quantum yield of CDs can reach as high as $33.8 \%$. Importantly, the CDs reveal great
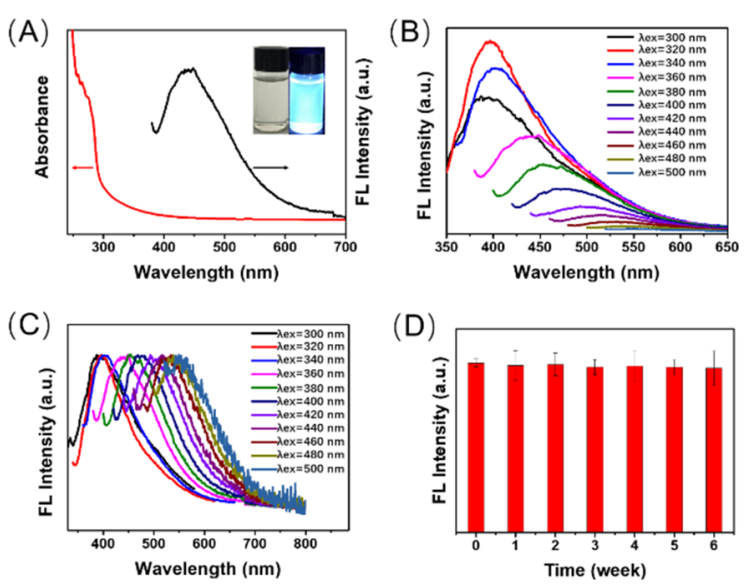

Figure 5. (A) UV-vis spectrum and FL spectrum of CDs; the inset showed the photographs of CDs in daylight and under UV light. (B) FL spectra of CDs with different excitation wavelengths, (C) the normalized FL spectra of CDs with different excitation wavelengths, and (D) the photostability of CDs during several weeks.

photostability, as depicted in Figure 5D, which can be stored for 6 weeks without an obvious decrement of luminescence intensity. These features make the CDs quite favorable for bioimaging applications.

2.3. Cytotoxicity of CDs in Cells. The cytotoxicity of prepared CDs was investigated with A549 cells via a cell counting kit-8 (CCK-8) assay. As demonstrated in Figure 6A,
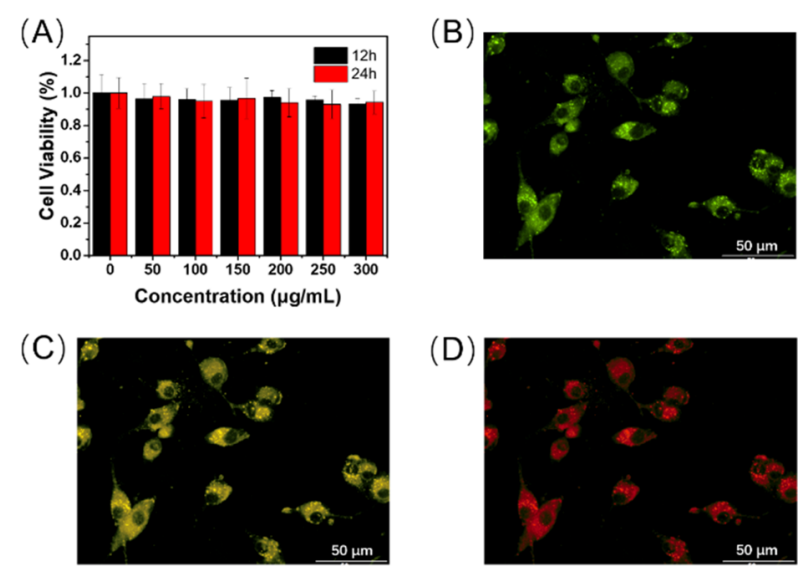

Figure 6. (A) Cell viability of A549 cells toward CDs with diverse concentrations in 12 and $24 \mathrm{~h}$ and the fluorescence imaging picture of A549 cells with $\lambda_{\text {ex }}$ of (B) 408, (C) 532, and (D) $633 \mathrm{~nm}$.

the cell viability of A549 cells remained with no obvious changes after different concentrations of CDs were put into the culture media of CDs for 12 and $24 \mathrm{~h}$. Even when the concentration of CDs reached as high as $300 \mu \mathrm{g} / \mathrm{mL}$, the viabilities of A549 cells remained more than $91 \%$ after $24 \mathrm{~h}$. These results proved the excellent biocompatibility of CDs for bioimaging and other bioapplications.

2.4. CDs for Celluar Imaging. Taking advantages of their favorable fluorescent properties and excellent biocompatibility, the cell imaging behavior of CDs was detected. The A549 cells were employed as model cells for the experiments. After the cells were incubated with CDs of $50 \mu \mathrm{g} / \mathrm{mL}$ for $3 \mathrm{~h}$, the cells were cleaned and exposed to fluorescence microscopy. As illustrated in Figure $6 \mathrm{~B}-\mathrm{D}$, the cytoplasm region of cells could be lightened with intense fluorescence of different colors as the 
excitation wavelengths changed, which demonstrated that the CDs can be potentially used for multicolor bioimaging of cells. Furthermore, it could be also observed that the morphology of A549 cells still remained well after incubation with CDs for 3 h. These results illustrated that the CDs were promising for bioimaging applications. ${ }^{34}$

2.5. CDs for Anti-Counterfeiting. Owing to their great photostability and tunable fluorescent properties, the obtained CDs can be potentially used as an anti-counterfeiting ink. As exhibited in Figure 7A, the water dispersion of CDs was

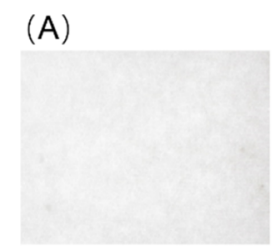

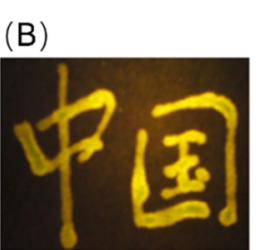

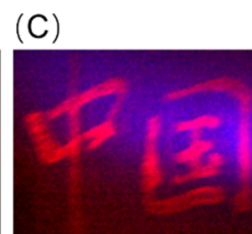

Figure 7. (A) Picture of a cellulosic paper in white light with characters written on them using CDs as an anti-counterfeiting ink, (B) picture of the above cellulosic paper under a laser irradiation of $532 \mathrm{~nm}$, and (C) picture of the above cellulosic paper under a laser irradiation of $633 \mathrm{~nm}$.

applied for writing two Chinese characters on the cellulosic papers, which were invisible under white light. After the cellulosic papers were exposed to 532 and $633 \mathrm{~nm}$ laser irradiation, the words converted to be visible as bright yellow and red Chinese characters (Figure $7 \mathrm{~B}$ and Figure $7 \mathrm{C}$, respectively). These experiments preliminarily proved the potential of CDs for anti-counterfeiting applications.

\section{CONCLUSIONS}

In one word, we have fabricated a facile and green strategy to rapidly construct $\mathrm{CDs}$ from gelatin. In this sonochemicalassisted approach, the nitrogen-doped CDs can be obtained through irradiation by intensive ultrasonic waves from ultrasonic probes in $30 \mathrm{~min}$. As far as we know, this is the first example of the rapid synthesis of nitrogen-doped CDs from gelatin. The achieved CDs exhibit excellent water dispersibility, remarkable fluorescent properties, high photostability, and negligible cytotoxicity. Furthermore, the CDs have been nicely utilized for fluorescence imaging of A549 cells and anti-counterfeiting applications. Significantly, the sonochemical-assisted approach to rapidly fabricate CDs is versatile for the facile construction of fluorescent CDs from many analogous carbon sources, and the obtained CDs can be potentially used in various areas including bioimaging and anticounterfeiting.

\section{EXPERIMENTAL SECTION}

4.1. Materials. Gelatin (type-B, 100 bloom), papain, sodium acetate, and acetic acid were received from Aladdin (Shanghai, China), all of which were analytical reagents. Reagents and consumables for cell experiments were all received from Invitrogen. (California, USA).

4.2. Rapid Preparation of CDs. Gelatin ( $1 \mathrm{~g}$ ) was dissolved in $200 \mathrm{~mL}$ of water at $50{ }^{\circ} \mathrm{C}$ under continuous stirring, and then, $30 \mathrm{mg}$ of papain together with several drops of acetic acid buffer ( $\mathrm{pH}$ 5.0) was put into the above water solution to hydrolyze the gelatin. After stirring for about 30 min, an ultrasonic probe was put below in the obtained solution, which was further irradiated with ultrasound for 30 min at $20 \mathrm{kHz}$ and $720 \mathrm{~W}$ (Scientz-2400F, Scientz, China). Then, the above solution was treated with centrifugation for 10 $\mathrm{min}$ at $12,000 \mathrm{rpm}$, and the supernatant containing CDs was separated by a filter membrane of $0.22 \mu \mathrm{m}$. The achieved solution of CDs can be stored at $4{ }^{\circ} \mathrm{C}$ or dried through further lyophilization. The yield of CDs was calculated to be $41.2 \%$.

4.3. Characterization of the CDs. The morphologies and microstructures of CDs were determined by HR-TEM of JEM2100F. FTIR and XPS of CDs were obtained by a Nicolet IS10 spectrometer (Nicolet, USA) and a Thermo ESCALAB 250Xi spectrometer (Thermo Fisher, USA), respectively. Their optical spectra were checked with a UV-3600 spectrometer (Shimadzu, Japan) and a Fls 1000/FS5 fluorophotometer (Edinburgh, England).

4.4. Cytotoxicity of the CDs. The cytotoxicity of CDs can be determined via testing their cell viability, which can be obtained through a CCK- 8 assay, and the detailed process can be found in our earlier works. ${ }^{30,35-38}$ First, A549 cells were carefully cultured in an incubator at $37{ }^{\circ} \mathrm{C}$ with $5 \% \mathrm{CO}_{2}$ to ensure their good growth. Before the CCK-8 test, A549 cells were transferred to microplates and cultured for $12 \mathrm{~h}$. Then, CDs in Dulbecco's modified Eagle's medium (DMEM) solution were put into each well for further incubation. After careful washing with phosphate buffer saline, the mixture of the CCK- 8 dye and culture medium was added to all the above wells and the microplates were put in an incubator for a further $2 \mathrm{~h}$. The optical density was detected at $480 \mathrm{~nm}$ with a Flex Station 3 microplate reader (Molecular Devices, USA).

4.5. Cellular Imaging. A549 cells were carefully cultured as described above before the imaging experiment, and A549 cells with a density of $1 \times 10^{5}$ cell $\mathrm{mL}^{-1}$ were maintained at 37 ${ }^{\circ} \mathrm{C}$ for $24 \mathrm{~h}$. Then, the A549 cells were transferred into a medium containing CDs of $50 \mu \mathrm{g} / \mathrm{mL}$ for $3 \mathrm{~h}$. After careful washes, the cells were imaged by an FV3000 confocal microscope (Olympus, Japan). For identifying their multicolor luminescence imaging behavior of CDs, different excitation wavelengths $(405,488$, and $633 \mathrm{~nm})$ were picked in these imaging experiments.

4.6. Anti-Counterfeiting. $\mathrm{CDs}$ in water $(0.5 \mathrm{mg} / \mathrm{mL})$ were prepared as an anti-counterfeiting ink, which was used for writing on a cellulosic paper. Then, the paper was exposed to lasers of 532 and $633 \mathrm{~nm}$, and their pictures were taken using a camera with the help of filters $(590 / 20 \mathrm{~nm}$ single-band bandpass filter and 660/13 nm single-band bandpass filter).

\section{ASSOCIATED CONTENT}

SI Supporting Information

The Supporting Information is available free of charge at https://pubs.acs.org/doi/10.1021/acsomega.0c05682.

High-resolution XPS scanning of sulfur (PDF)

\section{AUTHOR INFORMATION}

\section{Corresponding Authors}

Junjia Chen - Institute of Bioengineering, Guangdong Academy of Sciences, Guangzhou 510316, China; Guangdong Biomaterials Engineering Technology Research Center, Guangzhou 510316, China; Email: gzcsircjj@ 163.com

Ke Wang - Institute of Bioengineering, Guangdong Academy of Sciences, Guangzhou 510316, China; Guangdong Biomaterials Engineering Technology Research Center, 
Guangzhou 510316, China; @ orcid.org/0000-0002-6163-

4512; Email: wangke007@iccas.ac.cn

\section{Authors}

Chen Li - Institute of Bioengineering, Guangdong Academy of Sciences, Guangzhou 510316, China; Guangdong

Biomaterials Engineering Technology Research Center, Guangzhou 510316, China; ๑ orcid.org/0000-0002-75876408

Xiaoyan Sun - Institute of Bioengineering, Guangdong Academy of Sciences, Guangzhou 510316, China; Guangdong Biomaterials Engineering Technology Research Center, Guangzhou 510316, China

Yuan Li - Institute of Bioengineering, Guangdong Academy of Sciences, Guangzhou 510316, China; Guangdong Biomaterials Engineering Technology Research Center, Guangzhou 510316, China

Hailu Liu - Institute of Bioengineering, Guangdong Academy of Sciences, Guangzhou 510316, China; Guangdong Biomaterials Engineering Technology Research Center, Guangzhou 510316, China

Bibo Long - Institute of Bioengineering, Guangdong Academy of Sciences, Guangzhou 510316, China; Guangdong Biomaterials Engineering Technology Research Center, Guangzhou 510316, China

Dong Xie - Institute of Bioengineering, Guangdong Academy of Sciences, Guangzhou 510316, China; Guangdong Biomaterials Engineering Technology Research Center, Guangzhou 510316, China

Complete contact information is available at: https://pubs.acs.org/10.1021/acsomega.0c05682

\section{Notes}

The authors declare no competing financial interest.

\section{ACKNOWLEDGMENTS}

This research was supported by the GDAS' Project of Science and Technology Development (nos. 2020GDASYL20200102008 and 2018GDASCX-0105).

\section{REFERENCES}

(1) da Silva, J. C. G. E.; Gonçalves, H. M. R. Analytical and bioanalytical applications of carbon dots. TrAC, Trends Anal. Chem. 2011, 30, 1327-1336.

(2) Li, H.; He, X.; Kang, Z.; Huang, H.; Liu, Y.; Liu, J.; Lian, S.; Tsang, C. H. A.; Yang, X.; Lee, S.-T. Water-soluble fluorescent carbon quantum dots and photocatalyst design. Angew. Chem., Int. Ed. 2010, $49,4430-4434$

(3) Li, X.; Rui, M.; Song, J.; Shen, Z.; Zeng, H. Carbon and graphene quantum dots for optoelectronic and energy devices: a review. Adv. Func. Mater. 2015, 25, 4929-4947.

(4) Wang, X.; Cao, L.; Lu, F.; Meziani, M. J.; Li, H.; Qi, G.; Zhou, B.; Harruff, B. A.; Kermarrec, F.; Sun, Y.-P. Photoinduced electron transfers with carbon dots. Chem. Commun. 2009, 25, 3774-3776.

(5) Yan, F.; Jiang, Y.; Sun, X.; Wei, J.; Chen, L.; Zhang, Y. Multicolor carbon dots with concentration-tunable fluorescence and solventaffected aggregation states for white light-emitting diodes. Nano Res. 2020, 13, 52-60.

(6) Li, S.; Su, W.; Wu, H.; Yuan, T.; Yuan, C.; Liu, J.; Deng, G.; Gao, X.; Chen, Z.; Bao, Y.; Yuan, F.; Zhou, S.; Tan, H.; Li, Y.; Li, X.; Fan, L.; Zhu, J.; Chen, A. T.; Liu, F.; Zhou, Y.; Li, M.; Zhai, X.; Zhou, J. Targeted tumour theranostics in mice via carbon quantum dots structurally mimicking large amino acids. Nat. Biomed. Eng. 2020, 4, 704-716.
(7) Yan, F.; Bai, Z.; Zu, F.; Zhang, Y.; Sun, X.; Ma, T.; Chen, L. Yellow-emissive carbon dots with a large Stokes shift are viable fluorescent probes for detection and cellular imaging of silver ions and glutathione. Microchim. Acta 2019, 186, 113.

(8) Sankar, S.; Kaur, N.; Lee, S.; Kim, D. Y. Rapid sonochemical synthesis of spherical silica nanoparticles derived from brown rice husk. Ceram. Int. 2018, 44, 8720-8724.

(9) Huang, S.-W.; Lin, Y.-F.; Li, Y.-X.; Hu, C.-C.; Chiu, T.-C. Synthesis of fluorescent carbon dots as selective and sensitive probes for cupric ions and cell imaging. Molecules 2019, 24, 1785.

(10) Lauria, A.; Lizundia, E. Luminescent carbon dots obtained from polymeric waste. J. Cleaner Prod. 2020, 262, 121288.

(11) Peng, Z.; Ji, C.; Zhou, Y.; Zhao, T.; Leblanc, R. M. Polyethylene glycol (PEG) derived carbon dots: preparation and applications. Appl. Mater. Today 2020, 20, 100677.

(12) Sun, Y.-P.; Zhou, B.; Lin, Y.; Wang, W.; Fernando, K. A. S.; Pathak, P.; Meziani, M. J.; Harruff, B. A.; Wang, X.; Wang, H.; Luo, P. G.; Yang, H.; Kose, M. E.; Chen, B.; Veca, L. M.; Xie, S.-Y. Quantumsized carbon dots for bright and colorful photoluminescence. J. Am. Chem. Soc. 2006, 128, 7756-7757.

(13) Zhu, S.; Meng, Q.; Wang, L.; Zhang, J.; Song, Y.; Jin, H.; Zhang, K.; Sun, H.; Wang, H.; Yang, B. Highly photoluminescent carbon dots for multicolor patterning, sensors, and bioimaging. Angew. Chem., Int. Ed. 2013, 52, 3953-3957.

(14) Das, P.; Ganguly, S.; Agarwal, T.; Maity, P.; Ghosh, S.; Choudhary, S.; Gangopadhyay, S.; Maiti, T. K.; Dhara, S.; Banerjee, S.; Das, N. C. Heteroatom doped blue luminescent carbon dots as a nano-probe for targeted cell labeling and anticancer drug delivery vehicle. Mater. Chem. Phys. 2019, 237, 121860.

(15) Gonçalves, H.; da Silva, J. C. G. E. Fluorescent carbon dots capped with PEG200 and mercaptosuccinic acid. J. Fluoresc. 2010, 20, 1023-1028.

(16) Jia, X.; Li, J.; Wang, E. One-pot green synthesis of optically pHsensitive carbon dots with upconversion luminescence. Nanoscale 2012, 4, 5572-5575.

(17) Zhu, Z.; Cheng, R.; Ling, L.; Li, Q.; Chen, S. Rapid and largescale production of multi-fluorescence carbon dots by a magnetic hyperthermia method. Angew. Chem., Int. Ed. 2020, 59, 3099-3105.

(18) Kumar, R.; Kumar, V. B.; Gedanken, A. Sonochemical synthesis of carbon dots, mechanism, effect of parameters, and catalytic, energy, biomedical and tissue engineering applications. Ultrason. Sonochem. 2020, 64, 105009.

(19) Dehvari, K.; Liu, K. Y.; Tseng, P.-J.; Gedda, G.; Girma, W. M.; Chang, J.-Y. Sonochemical-assisted green synthesis of nitrogen-doped carbon dots from crab shell as targeted nanoprobes for cell imaging. J. Taiwan Inst. Chem. E.ng. 2019, 95, 495-503.

(20) Li, M.; Guo, Y.; Wei, Y.; MacDiarmid, A. G.; Lelkes, P. I. Electrospinning polyaniline-contained gelatin nanofibers for tissue engineering applications. Biomaterials 2006, 27, 2705-2715.

(21) Young, S.; Wong, M.; Tabata, Y.; Mikos, A. G. Gelatin as a delivery vehicle for the controlled release of bioactive molecules. $J$. Controlled Release 2005, 109, 256-274.

(22) Nezhad-Mokhtari, P.; Arsalani, N.; Ghorbani, M.; Hamishehkar, H. Development of biocompatible fluorescent gelatin nanocarriers for cell imaging and anticancer drug targeting. J. Mater. Sci. 2018, 53, 10679-10691.

(23) Baumgartner, M.; Hartmann, F.; Drack, M.; Preninger, D.; Wirthl, D.; Gerstmayr, R.; Lehner, L.; Mao, G.; Pruckner, R.; Demchyshyn, S.; Reiter, L.; Strobel, M.; Stockinger, T.; Schiller, D.; Kimeswenger, S.; Greibich, F.; Buchberger, G.; Bradt, E.; Hild, S.; Bauer, S.; Kaltenbrunner, M. Resilient yet entirely degradable gelatinbased biogels for soft robots and electronics. Nat. Mater. 2020, 19, $1102-1109$

(24) Tytgat, L.; Van Damme, L.; Van Hoorick, J.; Declercq, H.; Thienpont, H.; Ottevaere, H.; Blondeel, P.; Dubruel, P.; Van Vlierberghe, S. Additive manufacturing of photo-crosslinked gelatin scaffolds for adipose tissue engineering. Acta Biomater. 2019, 94, $340-350$. 
(25) Zhang, L.; Liu, J.; Zheng, X.; Zhang, A.; Zhang, X.; Tang, K. Pullulan dialdehyde crosslinked gelatin hydrogels with high strength for biomedical applications. Carbohydr. Polym. 2019, 216, 45-53.

(26) Liang, Q.; Ma, W.; Shi, Y.; Li, Z.; Yang, X. Easy synthesis of highly fluorescent carbon quantum dots from gelatin and their luminescent properties and applications. Carbon 2013, 60, 421-428.

(27) Wang, X.; Liu, H.; Wu, W.; Xiong, Y.; Chen, W.; Cao, K.; Wang, Y.; Chen, Y. One-step microwave approach to generate carbon dots/gelatin composite with both thermoresponsive sol-gel transition and fluorescence properties. Macromol. Mater. Eng. 2020, 305, 2000035.

(28) Abdollahi, A.; Herizchi, A.; Roghani-Mamaqani, H.; AlidaeiSharif, $\mathrm{H}$. Interaction of photoswitchable nanoparticles with cellulosic materials for anti-counterfeiting and authentication security documents. Carbohydr. Polym. 2020, 230, 115603.

(29) Ren, W.; Lin, G.; Clarke, C.; Zhou, J.; Jin, D. Optical Nanomaterials and Enabling Technologies for High-Security-Level Anticounterfeiting. Adv. Mater. 2020, 32, 1901430.

(30) Yan, F.; Sun, Z.; Zhang, H.; Sun, X.; Jiang, Y.; Bai, Z. The fluorescence mechanism of carbon dots, and methods for tuning their emission color: a review. Microchim. Acta 2019, 186, 583.

(31) Arsalani, N.; Nezhad-Mokhtari, P.; Jabbari, E. Microwaveassisted and one-step synthesis of PEG passivated fluorescent carbon dots from gelatin as an efficient nanocarrier for methotrexate delivery. Artif. Cells, Nanomed., Biotechnol. 2019, 47, 540-547.

(32) Dela Cruz, M. I. S.; Thongsai, N.; de Luna, M. D. G.; In, I.; Paoprasert, P. Preparation of highly photoluminescent carbon dots from polyurethane: Optimization using response surface methodology and selective detection of silver (I) ion. Colloids Surf., A 2019, 568, 184-194.

(33) Park, S. Y.; Lee, H. U.; Park, E. S.; Lee, S. C.; Lee, J.-W.; Jeong, S. W.; Kim, C. H.; Lee, Y.-C.; Huh, Y. S.; Lee, J. Photoluminescent green carbon nanodots from food-waste-derived sources: large-scale synthesis, properties, and biomedical applications. ACS Appl. Mater. Interfaces 2014, 6, 3365-3370.

(34) Wang, K.; Zhuang, J.; Liu, Y.; Xu, M.; Zhuang, J.; Chen, Z.; Wei, Y.; Zhang, Y. PEGylated chitosan nanoparticles with embedded bismuth sulfide for dual-wavelength fluorescent imaging and photothermal therapy. Carbohydr. Polym. 2018, 184, 445-452.

(35) Huang, H.; Cui, Y.; Liu, M.; Chen, J.; Wan, Q.; Wen, Y.; Deng, F.; Zhou, N.; Zhang, X.; Wei, Y. A one-step ultrasonic irradiation assisted strategy for the preparation of polymer-functionalized carbon quantum dots and their biological imaging. J. Colloid Interface Sci. 2018, 532, 767-773.

(36) Chen, J.; Cui, Y.; Liu, M.; Huang, H.; Deng, F.; Mao, L.; Wen, Y.; Tian, J.; Zhang, X.; Wei, Y. Surface grafting of fluorescent polymers on halloysite nanotubes through metal-free light-induced controlled polymerization: Preparation, characterization and biological imaging. Mater. Sci. Eng.: C 2020, 111, 110804.

(37) Wang, K.; Zhang, X.; Zhang, X.; Yang, B.; Li, Z.; Zhang, Q.; Huang, Z.; Wei, Y. Fabrication of cross-linked fluorescent polymer nanoparticles and their cell imaging applications. J. Mater. Chem. C 2015, 3, 1854-1860.

(38) Wang, K.; Fan, X.; Zhao, L.; Zhang, X.; Zhang, X.; Li, Z.; Yuan, Q.; Zhang, Q.; Huang, Z.; Xie, W.; Zhang, Y.; Wei, Y. Aggregation induced emission fluorogens based nanotheranostics for targeted and imaging-guided chemo-photothermal combination therapy. Small 2016, 12, 6568-6575. 